\title{
Tomographic-PIV measurement of the flow around a zigzag boundary layer trip
}

\author{
G. E. Elsinga $\cdot$ J. Westerweel
}

Received: 6 October 2010/Revised: 9 June 2011/Accepted: 23 June 2011/Published online: 8 July 2011

(C) The Author(s) 2011. This article is published with open access at Springerlink.com

\begin{abstract}
Tomographic-PIV was used to measure the boundary layer transition forced by a zigzag trip. The resulting instantaneous three-dimensional velocity distributions are used to quantitatively visualize the flow structures. They reveal undulating spanwise vortices directly behind the trip, which break up into individual arches and then develop into the hairpin-like structures typical of wallbounded turbulence. Compared to the instantaneous flow structure, the structure of the average velocity field is very different showing streamwise vortices. Such streamwise vortices are often associated with the low-speed streaks occurring in bypass transition flows, but in this case clearly are an artifact of the averaging. Rather, the present streaks in the separated flow region directly behind the trip are resulting from the waviness in the spanwise vortices as introduced by the zigzag trip. Furthermore, these streaks and the separated flow region are observed to be related to a large-scale, spanwise uniform unsteadiness in the flow that contributes significantly to the velocity fluctuations over large downstream distances (up to at least the edge of the present measurement domain).
\end{abstract}

\section{Introduction}

Boundary layer tripping, i.e., forcing it from a laminar state into a turbulent state, is commonly used to fix the point of transition, to prevent laminar separation bubbles from occurring and to reduce the drag of bluff bodies at certain Reynolds numbers. This forcing can be performed through

G. E. Elsinga $(\bowtie) \cdot$ J. Westerweel

Laboratory for Aero and Hydrodynamics,

Delft University of Technology, Delft, The Netherlands

e-mail: g.e.elsinga@tudelft.nl various means: blowing/suction through a slot in the wall, vibrating ribbons, and passive roughness elements attached to the wall such as sandpaper, 3-D roughness, rods, and zigzag strips. Out of this last group, the zigzag strip (illustrated in Fig. 1) is thought to be very efficient in terms of having the lowest Reynolds number based on roughness height that is required to initiate transition, namely 200 (Van Rooij and Timmer 2003), compared to 300-600 for the other cases (Braslow and Knox 1958). As a result, the zigzag strip can be of a smaller height than other 2-D strips, resulting in a reduction in the drag on the strip. However, when compared to 3-D grit roughness, the drag on the zigzag strip would be higher (Van Rooij and Timmer 2003). Due to the mentioned effectiveness of the zigzag strip, it is widely applied not only in wind tunnels but also on the wings of gliders and in sports (for instance, on the legs of speed skaters and oars of rowers to reduce the wake flow resulting in overall drag reduction). These trips, therefore, have an important engineering interest.

Generally, the associated path to turbulence is referred to as bypass transition (Reshotko 2007) due to the large initial disturbance introduced by the tripping device. Yet, it is not completely clear (1) what kind of flow structures are actually introduced by this specific trip, (2) how long they persist downstream, and (3) how they develop into canonical boundary layer turbulence with the well-known hairpin structures (Adrian 2007). Previous oil film surface flow visualizations behind zigzag trips (Lyon et al. 1997, Boermans 2006) have revealed backflow in small cellular regions immediately downstream of the upstream pointing spike, which are followed by clear oil stripes in the streamwise direction. These stripes have been associated in the past to streamwise vortices, which are considered to experience maximum spatial energy growth (Andersson et al. 1999) and then develop into turbulence (see also 


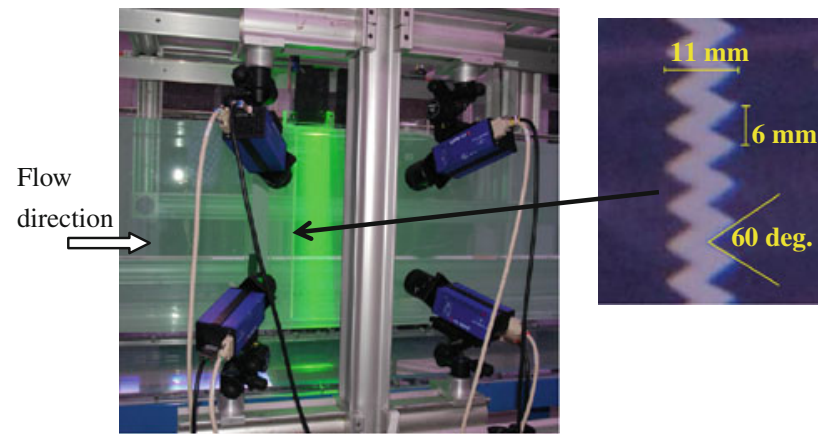

Fig. 1 Experimental arrangement in the water tunnel with detail of the zigzag trip

Swearingen and Blackwelder 1987). Such a scenario, if indeed correct, could explain an increased effectiveness of zigzag trip over other tripping devices.

In comparison, the flow structure behind 2-D tripping wires has received much more attention. Using dye or smoke flow visualization techniques, Hama et al. (1957) and Perry et al. (1981) observed spanwise structures separating from the wire, which were interpreted as spanwise vortices. When convecting downstream, these vortex lines developed an increasing spanwise waviness until breaking up into signatures of, what were believed to be, individual $\Lambda$-shaped vortices. In these studies, there was no evidence of streamwise vortices. Interestingly, streamwise vortices have again been reported behind vibrating ribbons attached to the wall with spanwise periodic spacers (Klebanoff et al. 1962) and pins (e.g., Fransson et al. 2004, Lavoie et al. 2008). Note that these observations were based on mean flow measurements using hot-wires. From these results, it may seem that such streamwise vortices are typical for 3-D trips rather than 2-D wires.

The bypass transition studies mentioned above report one common feature, which are elongated streaks of lowspeed flow. This applies to both tripping by roughness as well as by free-stream turbulence (e.g., Brandt et al. 2004, $\mathrm{Wu}$ and Moin 2009). However, the type of vortical structure (streamwise, spanwise or $\Lambda$ vortices) that is associated with these streaks differs in the various studies and may very well be more sensitive to the nature and strength of the initial forcing. Such sensitivity is also encountered in the closely related case of a transitional separation bubble, where the vortices in the separated shear layer may be either of Kelvin-Helmholtz type (Spalart and Strelets 2000 ) or nearly streamwise $\Lambda$ vortices (Alam and Sandham 2000).

Concerning the downstream effect of trips, further hotwire measurements by Erm and Joubert (1991) demonstrated the influence of several devices (wires, pins and distributed grit) on the velocity statistics. They showed that this influence reduces far downstream of the trip until it disappears and the velocity statistics return to their common values in a developed turbulent boundary layer.

In the present work, the instantaneous flow around a zigzag trip has been measured at different Reynolds numbers in order to elucidate the features of that specific type of forced boundary layer transition. The aim is to establish the actual transition scenario and compare it to proposed models and related flow cases. Based on the results, the reported enhanced effectiveness of these trips may be explained.

The employed experimental method is the tomographicPIV technique (Elsinga et al. 2006). The resulting instantaneous three-dimensional velocity fields yield the velocity statistics and allow visualizing the instantaneous flow structure, which will prove to be very different from the structure of the average velocity field. Further, the spatial development from the vortical structures introduced by the trip to the typical turbulent boundary layer structures will be shown, as well as the transition features that persist much longer affecting the flow over larger distances downstream. The latter are energetic very large-scale structures, which will be studied in a POD mode analysis.

\section{Experimental setup}

\subsection{Flow facility and model}

The experiments were performed in the water tunnel of the Laboratory for Aero and Hydrodynamics at Delft University of Technology (Fig. 1), which had a cross-section of $600 \times 600 \mathrm{~mm}^{2}$. The boundary layer was created over a flat Plexiglas plate with an elliptical leading edge placed vertically in the tunnel, where at the sides of the plate, the flow was bounded by the tunnel bottom wall and the free surface (i.e., the plate protrudes the air water interface). The zigzag trip was put $145 \mathrm{~mm}$ downstream of and parallel to the leading edge and had the following dimensions: the height was $1.6 \mathrm{~mm}$ from the wall, the width was $11 \mathrm{~mm}$ in the streamwise direction, and finally the pitch was $6.0 \mathrm{~mm}$ in the spanwise direction (Fig. 1). Furthermore, the zigzag top angle is 60 degrees. The trip is a tape that sticks directly onto the model surface and is available from Glasfaser Flugzeug-Service GmbH. Similar trips, mainly with a different height, are frequently used on gliders and in aerodynamic research (examples from Delft include Van Rooij and Timmer 2003 and Boermans 2006). The free-stream velocities $U_{e}$ considered in this study were $0.21,0.29$, and $0.53 \mathrm{~m} / \mathrm{s}$ with a free-stream turbulence intensity level below $0.5 \%$ in all cases (for additional details on the tunnel with model see also Schröder et al. 2008).

The $x, y, z$ system of coordinates, and associated $u, v$, $w$ velocity components, in this study is defined with respect 
to the transition trip, where $y$ is the distance to the wall, $z$ the coordinate along the trip, and $x$ the distance from the trip along the wall. At the measurement location, the freestream flow direction is tilted by approximately 5 degrees with respect to the trip resulting in a non-zero average spanwise velocity $w$ (i.e., cross-flow). It is possible that this cross-flow is caused by the difference in boundary conditions at both sides of the plate (solid wall and free surface) combined with the pressure field near the elliptical leading edge, which also results in static surface waves locally. Note that the $x$-direction will nonetheless be referred to as the streamwise direction. Although the tilt was unintended, it does represent a more realistic situation occurring in practical applications on, for instance, actual wings or bluff bodies.

Below, the velocities and distances are made dimensionless using the free-stream velocity, $U_{e}$, and the undisturbed laminar boundary layer thickness, $\delta_{0}$, at the trip location, $x_{0}=145 \mathrm{~mm}$. Assuming Blasius laminar velocity profiles, the thickness $\delta_{0}$ is estimated by (White 1991):

$\delta_{0}=5.0 x_{0} / \sqrt{\operatorname{Re}_{x 0}}$ with $\quad \operatorname{Re}_{x 0}=\frac{U_{e} x_{0}}{v}$

which results in $\delta_{0}=4.2,3.5$ and $2.6 \mathrm{~mm}$ for the freestream velocities in Table 1. Also shown in Table 1 is the estimated Reynolds number of the undisturbed boundary layer at the trip location, $R e_{\theta, o}$. These are well below the threshold for which a transitional or fully turbulent boundary layer may exist, i.e., $R e_{\theta}=162$ and 320 , respectively (Preston 1958). Therefore, to cause early transition, not only a disturbance but also an added momentum loss is required. The surface-mounted roughness, in this case the zigzag strip, provides these both by means of the flow structures introduced in its wake and its overall drag.

The current trip can, furthermore, be compared against a few common engineering criteria available in the literature for forcing turbulent boundary layer flow (Table 1). First, Braslow and Knox (1958) provide an empirical relation between the flow conditions in the undisturbed laminar boundary and the minimum roughness height, $k_{c r}$, needed for tripping it, which is based on extensive wind tunnel testing. In particular, the method employs a Reynolds number, $R e_{k}$, based on the flow velocity at the trip height, which has a critical value that depends on the roughness geometry (e.g., 2-D wires or isolated 3-D elements). The original work of Braslow and Knox contains a value of $R e_{k}$ for 2-D tripping wires $\left(R e_{k}=300\right)$, but not for zigzag strips. Other authors (Van Rooij and Timmer 2003), however, have suggested that the latter are more efficient, and hence they have proposed a lower critical Reynolds number for zigzag trips $\left(R e_{k}=200\right)$. Due to this uncertainty, the resulting minimum trip heights for both critical Reynolds numbers are listed in Table 1. It can be seen that for $U_{e}=0.21 \mathrm{~m} / \mathrm{s}$ the current trip is approximately at the design condition (slightly over- or under-tripping depending on the actual $R e_{k}$ used). At higher free-stream velocities, the current forcing is somewhat stronger than necessary.

An alternative method for wires is mentioned in the book by White (1991, p 386; after the work of Gibbings 1959). It simply considers the free-stream velocity (compared to the boundary layer velocity at the trip height before) when defining the critical Reynolds number for the roughness height. The resulting trip heights (Table 1) are clearly higher compared to the previous method, which is illustrative for the arbitrariness in defining and determining when transition would be effective.

The case with $U_{e}=0.21 \mathrm{~m} / \mathrm{s}$ most closely resembles the design criteria for turbulence forcing according to Braslow and Knox (1958); therefore, the focus in this paper will be on that condition. Moreover, the results from the other cases are qualitatively similar and quantitative differences mainly concern the downstream length over which the transition takes place (that is, shortening with increased forcing).

Table 1 Undisturbed laminar boundary layer thickness, $\delta_{0}$, Reynolds number based on the momentum thickness, $R e_{\theta, 0}$, at the trip position, Reynolds number based on the streamwise trip position with respect

to the leading edge, $R e_{x 0}$, and the critical trip heights, $k_{c r}$, according to three different methods for each free-stream velocity considered

\begin{tabular}{|c|c|c|c|c|c|c|}
\hline \multirow[t]{2}{*}{$U_{e}(\mathrm{~m} / \mathrm{s})$} & \multirow[t]{2}{*}{$\delta_{O}(\mathrm{~mm})$} & \multirow[t]{2}{*}{$R e_{\theta, o}(-)$} & \multirow[t]{2}{*}{$\operatorname{Re}_{x o}(-)$} & \multicolumn{3}{|l|}{$k_{c r}(\mathrm{~mm})$} \\
\hline & & & & (BK200) & (BK300) & (W850) \\
\hline 0.21 & 4.2 & 116 & $3.0 \cdot 10^{4}$ & 1.6 & 2.0 & 4.0 \\
\hline 0.29 & 3.5 & 136 & $4.2 \cdot 10^{4}$ & 1.2 & 1.6 & 2.9 \\
\hline 0.53 & 2.6 & 184 & $7.7 \cdot 10^{4}$ & 0.8 & 1.0 & 1.6 \\
\hline
\end{tabular}

The height of the trip in this study is $1.6 \mathrm{~mm}$ for all velocities

(BK200) method of Braslow and Knox (1958) using a critical Reynolds number $R e_{k}=200$, which is based on a characteristic velocity in the undisturbed laminar boundary layer at the height of the trip

(BK300) same as (1) but using $R e_{k}=300$

(W850) method in White (1991, p. 386) using a critical Reynolds number $R e_{k}=850$, which is based on the free-stream velocity 
Finally, the spanwise wavelength of the zigzag strip (i.e., $6.0 \mathrm{~mm}$ ) is taken to be in between the boundary layer thickness, $\delta_{0}$, and $2.8 \delta_{0}$ (Table 1), which represent the most energetic spanwise wavelength in the outer layer of a turbulent boundary layer (e.g., Elsinga et al. 2010) and the spanwise wave length experiencing maximum spatial energy growth in a laminar boundary layer according to the work of Andersson et al. (1999), respectively. These are the length scales expected to dominate the flow during and/or after transition, and the current trip acts within that range.

\subsection{Tomographic-PIV setup}

The tomographic system consisted of four cameras (LaVision Imager Pro X) with a 2,048 $\times 2,048$ pixels image format and 14-bit gray-scale dynamic range, which were mounted with Scheimpflug adapters and lenses (Nikkor) with a $f=60 \mathrm{~mm}$ focal length and a $f / 16$ aperture. The off-axis viewing angle was approximately 30 degrees in air, reducing to 22 degrees in water due to the changes in refractive index at the tunnel wall. Given the small lens aperture (and therefore large depth-of-field), no prisms were deemed necessary to correct for the effects due to refraction. The working fluid, which is water, was seeded with $56-\mu \mathrm{m}$ polyamide tracers up to a concentration equivalent to particle image density of 0.03 particles/pixel. These particles were illuminated by a dual-cavity frequency-doubled $200 \mathrm{~mJ} /$ pulse Nd:YAG laser in a 7-mm-thick sheet touching the wall. The total measurement volume located directly behind the trip was $120 \times 55 \times 7 \mathrm{~mm}^{3}$ in the streamwise, spanwise, and wall-normal direction, respectively, which was imaged at a resolution of 18.5 pixels $/ \mathrm{mm}$. The recording rate was constant at $2 \mathrm{~Hz}$, while the time separation between the light pulses was adjusted between runs to yield an approximate 20 pixels particle displacement in the free stream for the three velocities considered.

The instantaneous particle intensity distribution was reconstructed in 3-D space using the MART tomographic algorithm (Elsinga et al. 2006). Compared to the original images, the volume resolution was reduced to 15.7 voxels $/ \mathrm{mm}$ in order to reduce the memory requirements for the computation of the tomographic volume reconstruction. Image pre-processing (that is, background subtraction and Gaussian smoothing using a $3 \times 3$ pixel filter length) and volume self-calibration (Wieneke 2008) were applied to improve the reconstruction.

The particle displacement field was obtained from these reconstructed volumes using an iterative cross-correlation technique with multigrid and window deformation (Scarano and Riethmuller 2000). The final cross-correlation volume size was $28 \times 28 \times 28$ voxels corresponding to $1.8 \times 1.8 \times 1.8 \mathrm{~mm}^{3}$, which resulted in $258 \times 123 \times 17$ vectors per snapshot using $75 \%$ overlap of interrogation volumes between adjacent correlation positions. At each flow condition, a dataset consisting of 1,000 of such velocity snapshots was acquired.

The current spatial resolution is comparable to the trip height and emphasizes the larger scales of motion in the transition. However, owing to the limited Reynolds number at the trip location $\left(R e_{k} \sim 200\right)$, much smaller energetic flow scales are not expected to be present.

The suitability of the tomographic-PIV technique for the study of coherent structures in wall-bounded flow has already been established previously (Elsinga 2008, Elsinga et al. 2010). The uncertainty of the particle displacement in these measurements has been estimated at approximately 0.2 pixels, which was found to apply here as well. The uncertainty was assessed based on an analysis of the divergence in the measured velocity fields (for details on this method we refer to the book by Adrian and Westerweel 2011).

\section{Results}

\subsection{Average velocity field}

The average flow is obtained by averaging the 1,000 snapshots of the velocity field, and the result for $U_{e}=0.21 \mathrm{~m} / \mathrm{s}$ is displayed in the Figs. 2, 3, 4. The downstream development of the flow in terms of its velocity components is firstly shown in a wall-parallel plane at $y / \delta_{O}=0.28$ (Fig. 2). All components reveal streak-like structures directly behind the trip, extending to about $x / \delta_{0}=5$. At the present distance from the wall, they are characterized by backflow (as evidenced by a negative $u$-component of the velocity) and a flow away from the wall (as evidenced by a positive $v$-component of the velocity) inside the streaks that start from the downstream pointing tips of the zigzag trip. Associated with the upstream pointing tips is a region of positive, but small, $u$ with $v<0$. The spanwise velocity component, $w$, is negative everywhere, but varies in magnitude, being largest inside the streaks starting from the downstream pointing tips. Following the streaks is a region where the average flow regains spanwise uniformity, accelerates spatially (i.e., $\partial u / \partial x>0$ ), and is directed toward the wall (i.e., $v<0)$. This region covers approximately $x / \delta_{0}=5-15$. Then, starting from $x / \delta_{0}=15$, the average flow is parallel to the wall (i.e., $v \approx 0$ ) with little variation in both the spanwise and the streamwise directions, as expected for regular (turbulent) boundary flow. As mentioned, the trip is not perfectly aligned normal to the free-stream flow, but rather is slightly tilted by about 5 degrees. As a result, some cross-flow $(w<0)$ is found, even far downstream of the trip. Moreover, the streaks are also tilted with respect to the 

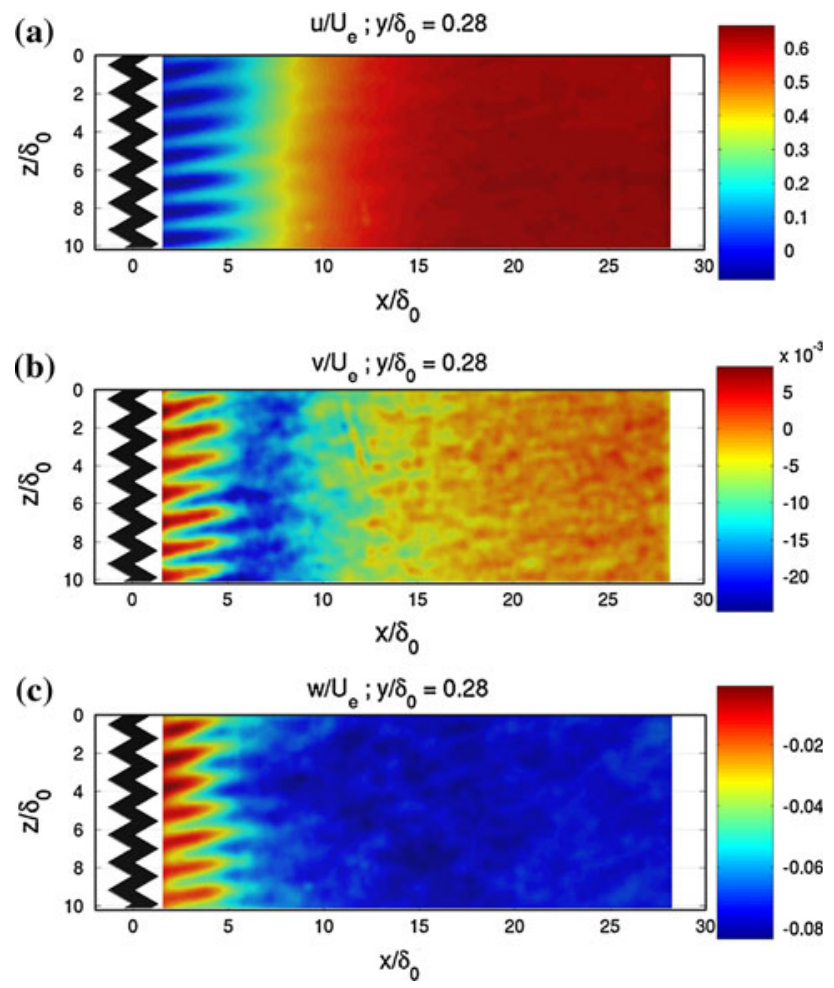

Fig. 2 Mean flow velocity in a plane parallel to the wall at $y / \delta_{0}=0.28\left(U_{e}=0.21 \mathrm{~m} / \mathrm{s}\right)$. a Streamwise velocity, b wall-normal velocity and $\mathbf{c}$ spanwise velocity component

$x$-axis, which has been defined based on the orientation of the trip and $n o t$ the free-stream velocity (which differ by 5 degrees).

A three-dimensional rendering of the average velocity field near the trip is presented in Fig. 3a. The blue isosurface marks a region of backflow $(u<0)$ immediately following the trip, which varies both in height and in length in the spanwise direction following the periodicity of the zigzag. This variation is visible in Fig. 2 as the streaks. Roughly above each streak is a streamwise vortex as detected by the $Q$-criterion (Hunt et al. 1988), shown in green in Fig. 2. These vortices are co-rotating and are associated with a swirling motion in the spanwise-wallnormal plane (Fig. 3b) that brings high-speed fluid toward the wall and transports low-speed (and backflow) fluid away from the wall. The lack of symmetry, in the sense that there are no counter-rotating vortex pairs, is again attributed to the 5-degree tilt of the trip causing a unidirectional cross-flow in the boundary layer behind it, which is known to contain co-rotating streamwise vortices (e.g., White and Saric 2005). As mentioned above, further downstream of the separated flow region, the average flow is uniform in the spanwise direction and therefore no longer contains three-dimensional features.

The average velocity vectors in the streamwise-wallnormal plane (Fig. 4) show a shear layer, probably emanating from the trailing edge of the trip, separating the reversed flow near the wall from the high-speed outer flow. In the velocity profiles, this introduces an inflection point, which is well known to be unstable and a source for creating turbulence. Due to the limited spatial resolution in the present experiments, it is very likely that the shear layer is smeared out and appears thicker than it actually is. Yet, its presence can be detected. The shear layer subsequently increases in thickness downstream, while the average flow reattaches and a turbulent boundary layer profiles starts to develop. At other spanwise locations, the flow development appears very similar.

\subsection{Reynolds stresses}

The Reynolds normal stresses $\left\langle u^{\prime} u^{\prime}\right\rangle,\left\langle v^{\prime} v^{\prime}\right\rangle$ and $\left\langle w^{\prime} w^{\prime}\right\rangle$ and the shear stress $\left\langle u^{\prime} v^{\prime}\right\rangle$ are presented in a streamwise-wallnormal plane through a downstream pointing tip (Fig. 5). The overall patterns and stress levels are similar at other spanwise positions. As expected, the shear layer separating from the zigzag trip is associated with high levels of $\left\langle u^{\prime} u^{\prime}\right\rangle$ and $\left\langle u^{\prime} v^{\prime}\right\rangle$. The peak normal stress $\left\langle u^{\prime} u^{\prime}\right\rangle$ progressively moves closer to the wall, while the peak shear stress $\left\langle u^{\prime} v^{\prime}\right\rangle$ remains at approximately the same height. The wall- (a)

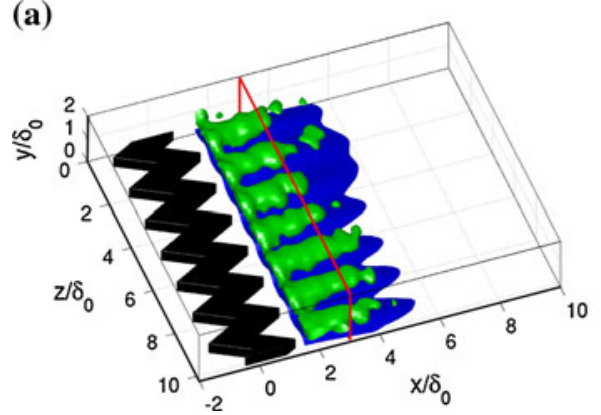

Fig. 3 a Three-dimensional rendering of the mean velocity field showing backflow (blue) and vortical motion as detected by the Q-criterion (green). b Velocity vectors in the cross-plane $x / \delta_{0}=3.05$ with contour of in-plane swirling strength (Zhou et al. 1999)

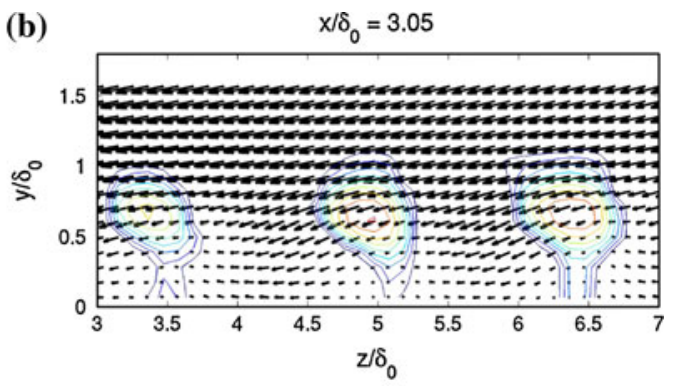

indicating the co-rotating vortices. Note that the velocity in the cores identified by the swirling strength is not zero so that swirling motion is combined with a convective velocity. The location of the plane in (b) is also indicated in (a) by the red lines 


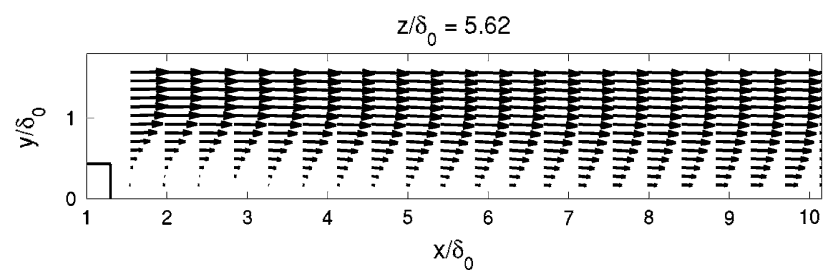

Fig. 4 Velocity vectors in the streamwise-wall-normal plane $z / \delta_{0}=5.62$ showing the separation bubble and reattachment behind the trip, which trailing edge is indicated by the rectangle in the lower left corner

normal stress $\left\langle v^{\prime} v^{\prime}\right\rangle$ increases strongly after the average flow reattaches $\left(x / \delta_{0}=5\right)$ with the peak values first at about the height of the trip and then slowly moving away from the wall. Similarly, the spanwise stress $\left\langle w^{\prime} w^{\prime}\right\rangle$ increases rapidly near the wall after average reattachment with the peak then moving away from the wall, but at a lower rate compared to $\left\langle v^{\prime} v^{\prime}\right\rangle$. From these results, it is clear that the redistribution of turbulent kinetic energy of the different components takes place in the region near the point of average reattachment, where the turbulence is strained by the flow moving toward the wall.
The spanwise stress $\left\langle w^{\prime} w^{\prime}\right\rangle$ also shows high values above the shear layer directly behind the trip $\left(\left\langle w^{\prime} w^{\prime}\right\rangle /\right.$ $\left.U_{e}^{2} \approx 5 \cdot 10^{-3}\right)$. This is associated with the unsteadiness in the separated flow region behind the trip, which displays large-scale streamwise and spanwise modes in a POD analysis, as will be demonstrated in Sect. 3.5 (the streamwise and spanwise flow is coupled to some extend due to the present cross-flow).

The present Reynolds stress levels after average reattachment are clearly much higher than those in a canonical developed turbulent boundary layer, which are approximately $2.5 \cdot 10^{-3}, 1.1 \cdot 10^{-3}, 1.6 \cdot 10^{-3}$, and $-0.8 \cdot 10^{-3}$ for $\left\langle u^{\prime} u^{\prime}\right\rangle / U_{e}^{2},\left\langle v^{\prime} v^{\prime}\right\rangle / U_{e}^{2},\left\langle w^{\prime} w^{\prime}\right\rangle / U_{e}^{2}$, and $\left\langle u^{\prime} v^{\prime}\right\rangle / U_{e}^{2}$, respectively, at $y / \delta=0.5$ (Klebanoff 1955). For example, $\left\langle u^{\prime} u^{\prime}\right\rangle$ and $\left\langle u^{\prime} v^{\prime}\right\rangle$ are amplified by a factor 7-10 at a distance of three boundary layer thicknesses downstream of average reattachment $\left(x / \delta_{0} \approx 8\right.$ in Fig. 5), which is in agreement with the enhancement of the Reynolds stresses downstream of a laminar separation, as reported by Castro and Epik (1998). Moreover, the order of magnitudes of the Reynolds stresses is consistent with results obtained just behind a turbulent separation (Alving and Fernholz 1996).
Fig. 5 Reynolds normal stresses $(\mathbf{a}-\mathbf{c})$ and Reynolds shear stress $\left\langle u^{\prime} v^{\prime}\right\rangle$ (d) in a streamwise-wall-normal plane at $z / \delta_{0}=5.62\left(U_{e}=0.21 \mathrm{~m} / \mathrm{s}\right)$. a streamwise, b wall-normal and $\mathbf{c}$ spanwise component
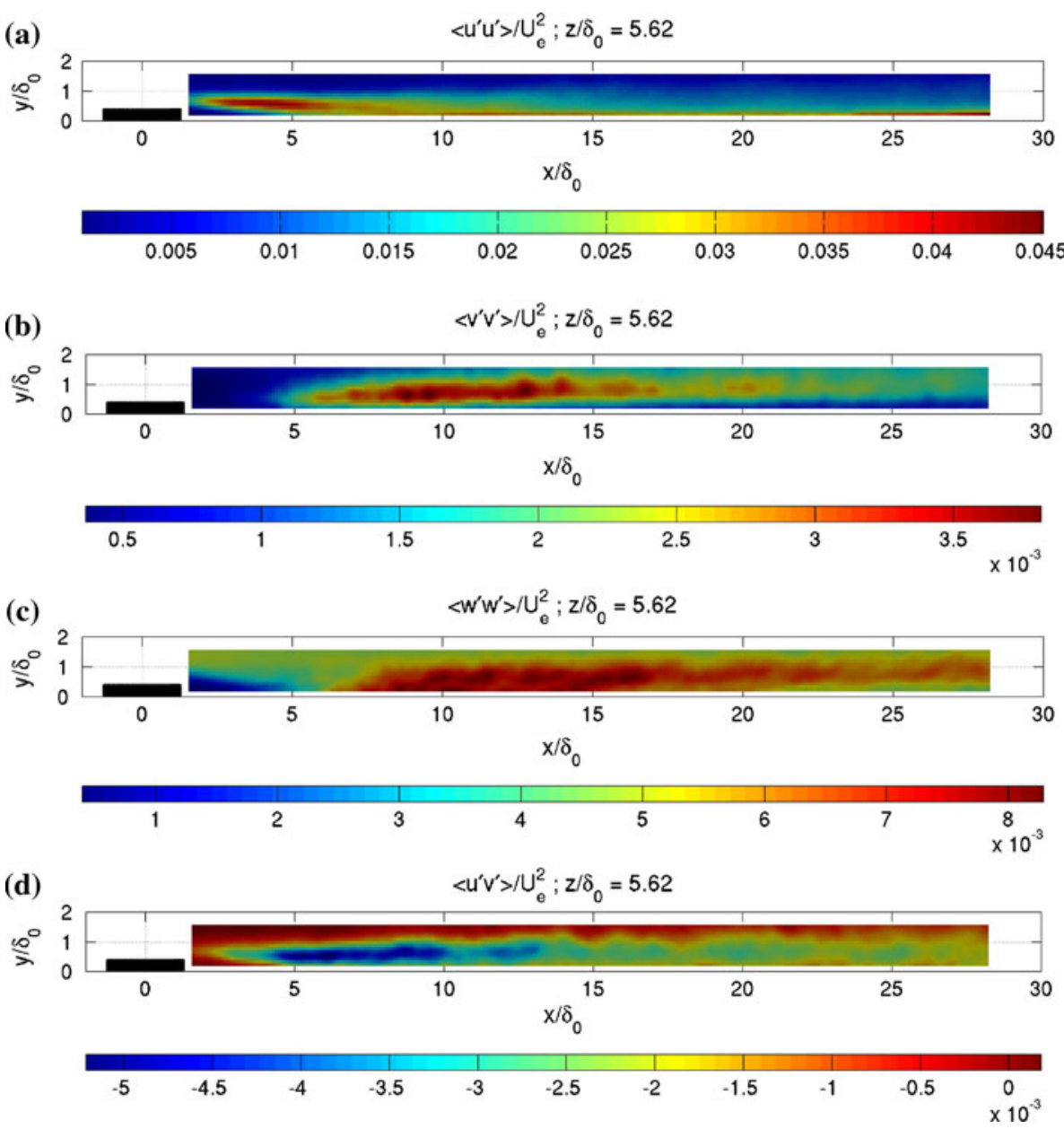

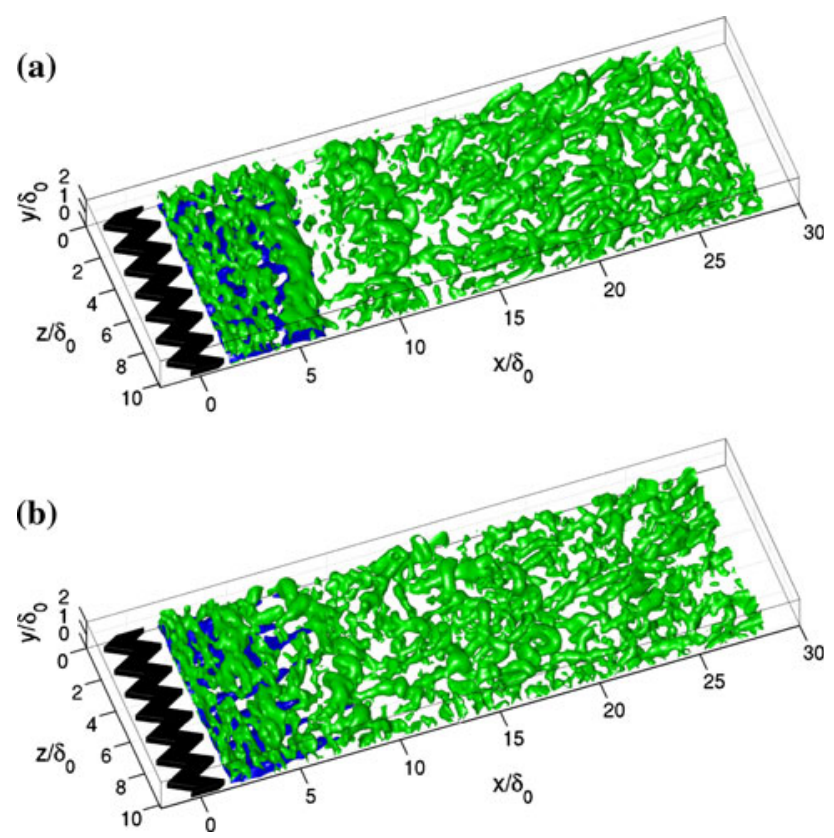

Fig. 6 Two sample instantaneous result for $U_{e}=0.21 \mathrm{~m} / \mathrm{s}$. Green indicates vortical motion using the Q-criterion and blue reveals backflow near the trip

The fact that the overall level of each Reynolds stress is higher than expected for a canonical turbulent boundary layer, and that these levels are still decreasing at the downstream edge of the measurement domain $\left(x / \delta_{0}=28\right)$, indicates that the boundary layer remains affected by the trip at least up to there. How this is reflected in the instantaneous turbulent structures will be shown below.

\subsection{Instantaneous flow fields}

Two typical results for the case with a free-stream velocity of $U_{e}=0.21 \mathrm{~m} / \mathrm{s}$ are shown in Fig. 6, displaying regions (in green) of vortical motion as identified by means of the $Q$-criterion (Hunt et al. 1988) and negative streamwise velocity (in blue). Immediately behind the trip, the flow is separated and a shear layer forms from the top of the trip similar to what has been observed for the average flow (Fig. 7). The instantaneous results (Figs. 6, 7, 8, 9) reveal also a very important difference with respect to the mean. Within the shear layer, vorticity rolls up into spanwise swirling motions rather than the streamwise vortices in the average velocity field. Due to the zigzag shape of the trip, these vortices undulate in the spanwise direction and have a small streamwise component to it (compare the contour levels of swirling strength computed from the in-plane velocities in Figs. 7 and 8). The latter component builds up coherently when averaging due to streamwise vortices traveling in the streamwise direction. On the other hand, the wall-normal velocity fluctuations, associated with the

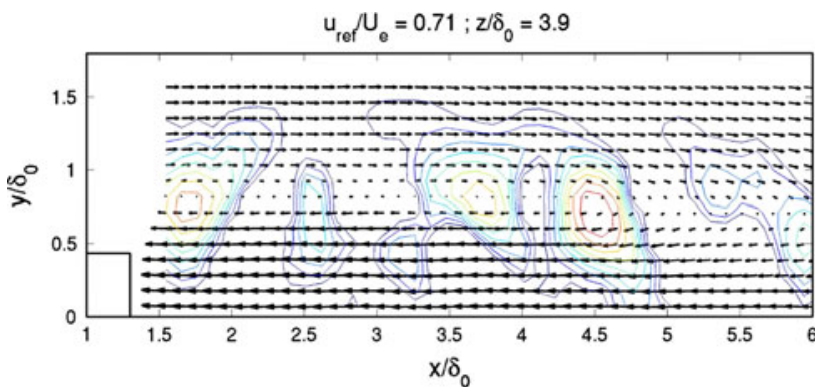

Fig. 7 Velocity vectors in the streamwise-wall-normal plane $z / \delta_{0}=3.9$ of the volume presented in Fig. $6 \mathrm{~b}$. The vectors are relative to a convective velocity $u_{\text {ref }}$ and show the strong separation shear layer containing swirling motion, which is detected using the in-plane swirling strength (spanwise swirling, contours). The trailing edge is indicated by the rectangle in the lower left corner

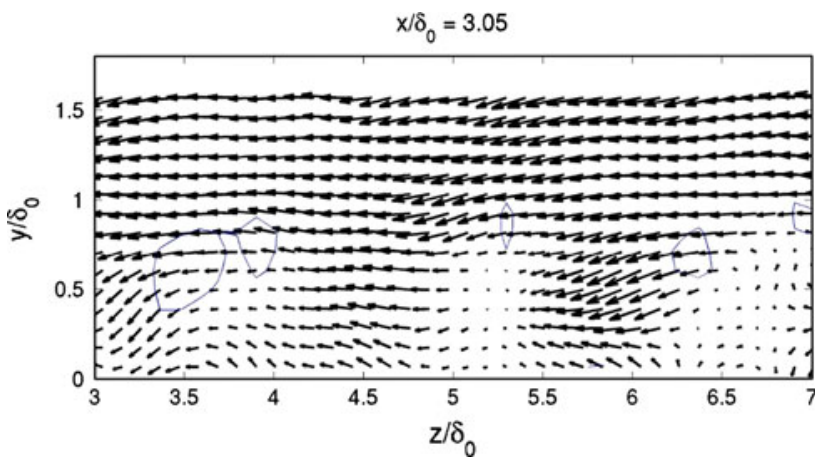

Fig. 8 Instantaneous velocity vectors in the cross-plane $x / \delta_{0}=3.05$ of the volume presented in Fig. $6 \mathrm{~b}$. The contours represent the inplane swirling strength (streamwise swirling). Note that the contour levels are identical to those in Fig. 7, but the vector length is rescaled. The swirling strength is clearly less than in the streamwise-wallnormal plane

spanwise vortices in a shear layer, tend to cancel each other when they convect downstream. The positive wall-normal fluctuation on one side of the vortex core cancels the negative fluctuation on the other side when it has moved by the vortex diameter. Averaging the velocity fields actually obscures the true nature of the instantaneous vortices and the turbulent production processes that can be associated with them. Therefore, having instantaneous 3-D measurements of the flow structure proves vital here, and we may speculate that this applies to other transition types as well.

At a downstream location of around $x / \delta_{0}=5$ (see Fig. 6), the spanwise vortices have broken up into individual arches that remain aligned, to some degree, in the spanwise direction (see also the detail in Fig. 9a). Because the necks of these structures are inclined at about 45 degrees with the wall, this process is associated with increasing wall-normal velocity fluctuations, visible as increasing levels of wall-normal Reynolds stress $\left\langle v^{\prime} v^{\prime}\right\rangle$ and 

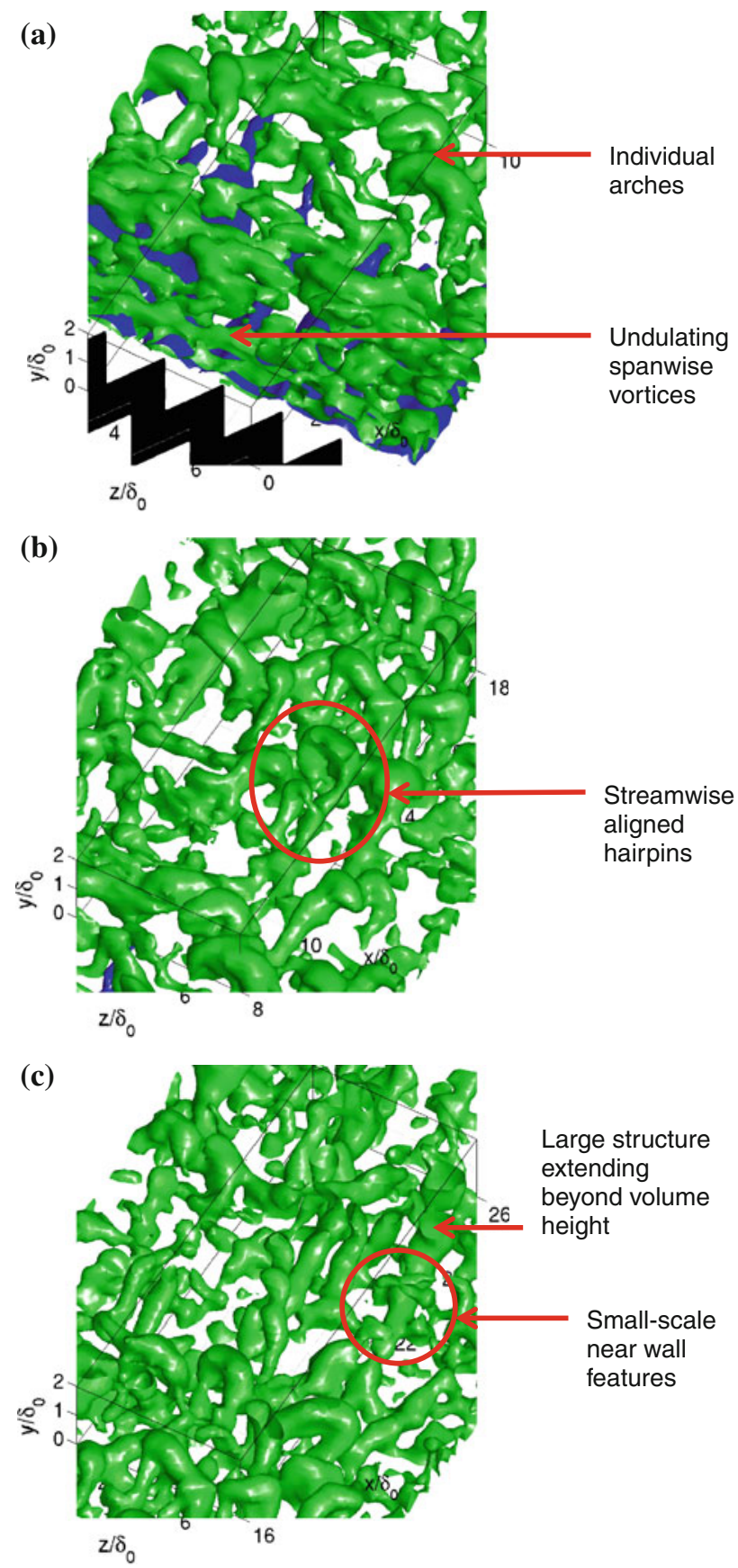

Fig. 9 Details of Fig. 6b near the trip a between $x / \delta_{0}=0$ and 10 , b between $x / \delta_{0}=8$ and 18 and $\mathbf{c}$ between $x / \delta_{0}=16$ and 26

Reynolds shear stress $\left\langle u^{\prime} v^{\prime}\right\rangle$ in the same region of the flow (Fig. 5).

Then, from the arches, hairpin-like (or cane-like) structures are seen to develop near $x / \delta_{0}=10$ (Fig. 9b), meaning that the arches now show legs, which are quasistreamwise vortices connected to the necks of the arches (Robinson 1991). They are related to the stretching of streamwise vorticity due to the accelerating flow in that region $(\partial u / \partial x>0$, see Sect. 3.1). Streamwise oriented vortices correspond to spanwise and wall-normal velocity fluctuations, and as a result, both the Reynolds normal stresses $\left\langle v^{\prime} v^{\prime}\right\rangle$ and $\left\langle w^{\prime} w^{\prime}\right\rangle$ grow (Fig. 5). Also visible here is the streamwise alignment of a number hairpins forming a so-called packet (Adrian et al. 2000). One example of which is indicated in Fig. 9b. It is noted that other type (undefined) vortex structures are observed as well (Fig. 9b).

The range of flow scales is limited at this stage (i.e., the vortices are all of similar size), but this changes downstream toward the end of the present measurement domain $\left(x / \delta_{0}>18\right.$, Fig. 9c). There, structures covering the complete height of the measurement volume (likely extending beyond it) are seen, as well as new small-scale near-wall features. It is speculated that these small scales are linked to the well-known near-wall low-speed streaks in fully developed turbulent boundary layers (Kline et al. 1967), which form in this region and which explain the increasing value of $\left\langle u^{\prime} u^{\prime}\right\rangle$ very close to the wall that can be seen in Fig. 5 for $x / \delta_{0}>22$. The characteristics of small-scale near-wall turbulence developing well downstream of reattachment is consistent with observations made in a transitional separation bubble (Alam and Sandham 2000) and the slow redevelopment of the inner layer after a fully turbulent separation bubble (Alving and Fernholz 1996).

Some of the features in Fig. 9 are reminiscent of previously proposed models describing the generation of vortical structures in wall-bounded turbulence. In particular, the example of a larger hairpin leg with associated small-scale vortices (Fig. 9c) may be associated with the generation of vortices via surface interaction as proposed by Smith et al. (1991). In brief, the leg induces a lowspeed region near the wall with an adverse pressure gradient, which in turn becomes unstable and forms a sheet that rolls up into a new near-wall hairpin adjacent to the existing leg. Furthermore, the packet of similar size hairpins in Fig. 9b is suggestive of the auto-generation model (Zhou et al. 1999), where the primary hairpin of sufficient strength generates a secondary upstream, which grows to a similar size as the primary thereby forming a coherent vortex packet. The visualizations give some support to the idea that these mechanisms may be at work here, although temporal information would be needed to confirm this.

\subsection{Spanwise coherence}

From the visualization of vortical structures, it may appear that the original spanwise alignment of arches (Fig. 9a) gradually disappears further downstream (Fig. 9c). While the individual structures may be characteristic of a developed turbulent boundary layer (that is, hairpins and 


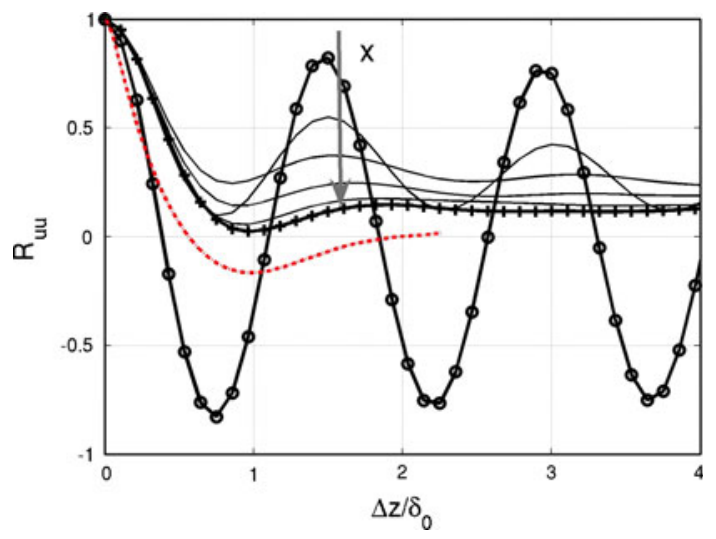

Fig. 10 Autocorrelation coefficient in the spanwise direction of the fluctuating $u$-component of velocity with respect to the ensemble, spanwise average. The profiles (black) are taken at $y / \delta_{0}=0.39$ and for $x / \delta_{0}=2,7,12,17,22$, and 27, where the first and last location are labeled with $o$ and + , respectively. Increasing $x$ position is also indicated by the gray arrow. For comparison, the spanwise autocorrelation in the turbulent boundary layer $2 \mathrm{~m}\left(\sim 500 \delta_{0}\right)$ downstream of the trip is given in red (dash-dot line). Note that the spanwise coordinate in the latter case has been rescaled to fit the graph

packets), there remains an important spanwise coherence in the flow, which must be ascribed to the tripping. It was already noted that the Reynolds stresses (Sect. 3.2) indicate that the flow is still affected by the transition at $x / \delta_{0}=28$.

The spanwise coherence of the flow can be demonstrated by considering the spanwise spatial autocorrelation of the fluctuating $u$-component of the velocity taken at a height $y / \delta_{0}=0.39$. Here, the fluctuations are taken with respect to the average over the ensemble of snapshots and over the spanwise direction. The additional averaging over the spanwise direction only affects the results up to $x / \delta_{0}=7$, after which the ensemble average is uniform in that direction (Fig. 2a). The resulting profiles for the autocorrelation coefficient at various $x$ locations are presented in Fig. 10. The first profile $\left(x / \delta_{0}=2\right)$ clearly shows the dominating spanwise oscillations corresponding to the streaks directly behind the trip. The height of the correlation peaks drops slowly, suggesting a large spanwise coherence between the streaks as also observed in the instantaneous snapshots (Figs. 6 and 9a). The spanwise oscillation is then seen to reduce in amplitude with increasing $x$, while at the same time a positive plateau in the autocorrelation coefficient gradually forms for $\Delta z / \delta_{0}>2$. The plateau and the fact that the autocorrelation coefficient does not drop below zero is evidence for a large spanwise coherence or spanwise alignment of structures in the flow, which exists in the flow at least up to $x / \delta_{0}=27$ where the plateau autocorrelation coefficient is still relatively high at 0.15 . It is regarded as the reminiscent of the spanwise vortices in the shear layer coming off the trip at the early stages of transition (Sect. 3.3) that is also responsible for the relatively high values for the Reynolds stresses throughout the current measurement domain (Sect. 3.2).

Before reaching a plateau, the autocorrelation coefficient has a minimum value with a negative correlation coefficient near $\Delta z / \delta_{0}=1$, which is associated with the typical size of the spanwise variations in the streamwise velocity, similar to the high- and low-momentum zones in the outer layer of developed turbulent boundary layers (e.g., Hutchins and Marusic 2007, Elsinga et al. 2010). However, in comparison, the autocorrelation profile expected for a developed turbulent boundary layer (the red dashed-dot line in Fig. 10) reaches a distinctly different plateau at zero. The developed turbulent boundary layer profile used here has been obtained $2 \mathrm{~m}\left(\begin{array}{cc}\sim 500 & \delta_{0}\end{array}\right)$ downstream of the trip in the same flow facility, where velocity data were acquired by the same 4-camera tomographic-PIV system. The measured autocorrelation profile agrees well with those available in the literature for other developed turbulent boundary layers (e.g., Hutchins and Marusic 2007), from which it can be concluded that the transition structures no longer affect the boundary layer at a distance of $500 \delta_{0}$ downstream of the trip. Moreover, at that location the profiles of the Reynolds stresses have returned to the shape and amplitudes expected for a developed turbulent boundary layer (Schröder et al. 2008). It is important to realize that the transitional structures are no longer affecting the boundary layer characteristics at the aforementioned location far downstream of the trip, as this was used in some earlier experimental studies of timeresolved 3-D velocity data (Schröder et al. 2008, Elsinga and Marusic 2010). The transitional structures are likely to disappear at shorter distances from the trip, but at present no measurements have been performed at intermediate locations, so this point remains open for now.

\subsection{Large-scale energetic modes}

Further investigation into the large-scale modes in the flow is performed using proper orthogonal decomposition (POD, Berkooz et al. 1993). This technique decomposes the ensemble of fluctuating velocity fields into a set of uncorrelated basis functions, or modes, which do need to be specified beforehand and are ordered according to their turbulent kinetic energy content. It can be shown that such decomposition is the most efficient; meaning that for a given number of modes POD captures more of the turbulent signal's energy than any other set of basis functions in fixed coordinates. That is, a limited number of modes are needed to describe the flow up to a certain energy content. The particular scheme employed here is the method of snapshots (Sirovich 1987; Humble et al. 2007).

The resulting first two POD modes appear relatively strong containing 13 and 9 percent of the total turbulent 


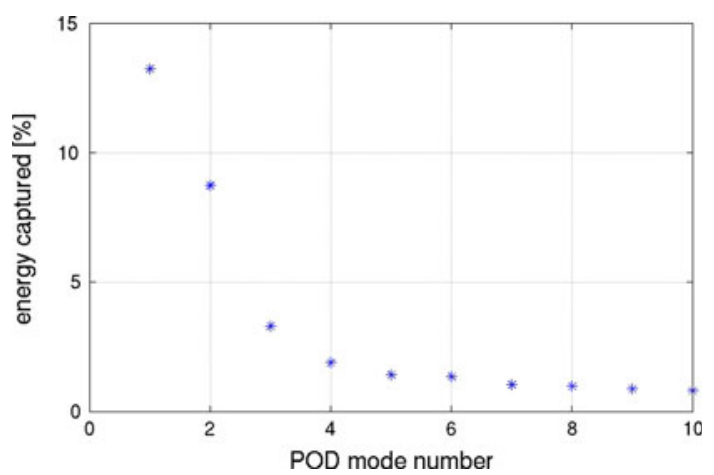

Fig. 11 Percentage of the total turbulent kinetic energy captured by each POD mode

kinetic energy (Fig. 11) compared to only one percent for mode 5 and higher. The fluctuating velocity field of the first POD mode (Fig. 12) reveals elongated regions of spanwise alternating positive and negative $u$ ' close to the trip, which are associated with negative and positive wall-normal velocity fluctuations, respectively. These elongated regions are, in the spanwise direction, located adjacent to the central axis of each streak observed in the average velocity field (Fig. 2). Furthermore, the $u^{\prime}$ peak value is attained not directly behind the trip, but rather somewhat downstream at $\Delta x / \delta_{0}=4$. Hence, adding or subtracting this mode from the average flow field has the effect of redirecting/tilting the streaks behind the trip. Because this tilting is approximately the same for all streaks, it can be considered a large-scale unsteadiness of the flow.

The second POD mode represents a nearly spanwise uniform transport of high momentum fluid toward the wall. The fluctuating velocity of this mode (Fig. 13) shows fluid moving toward the wall $\left(v^{\prime}<0\right)$ around $\Delta x / \delta_{0}=6$, which coincides with the location of average flow reattachment (Sect. 3.1). The result is an increase in the streamwise velocity downstream at $\Delta x / \delta_{0}=10\left(u^{\prime}>0\right)$ and negative $u^{\prime}$ in the streaks near the wall upstream at $\Delta x / \delta_{0}=4$. The latter causes an increasing backflow velocity in these streaks with respect to the average flow. Furthermore, the positive $u^{\prime}$ fluctuating velocity decreases toward the downstream edge of the measurement domain, but remains significant. The second POD mode, like the first, represents an energetic and very large-scale coherent fluctuation, which is associated with the dynamics of the flow features introduced by the transition trip, because of the location of the fluctuating velocity peaks. In particular, the second mode, when combined with the average flow, changes the size of the separation bubble and the point of reattachment of the separated shear layer. Furthermore, the downstream extend of significant $u^{\prime}$ in the second mode shows that these features of the trip transition affect the flow even beyond the current measurement domain.
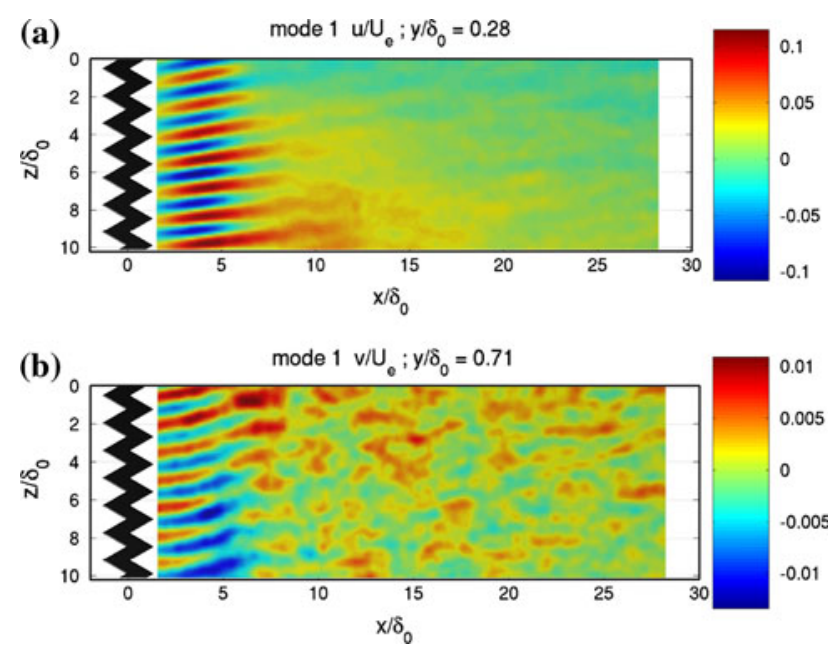

Fig. 12 POD mode 1 associated a tilting of the streaks directly behind the trip, a $u^{\prime}$-component and $\mathbf{b} v^{\prime}$-component in wall-parallel planes $y / \delta_{0}=0.28$ and 0.71 , respectively
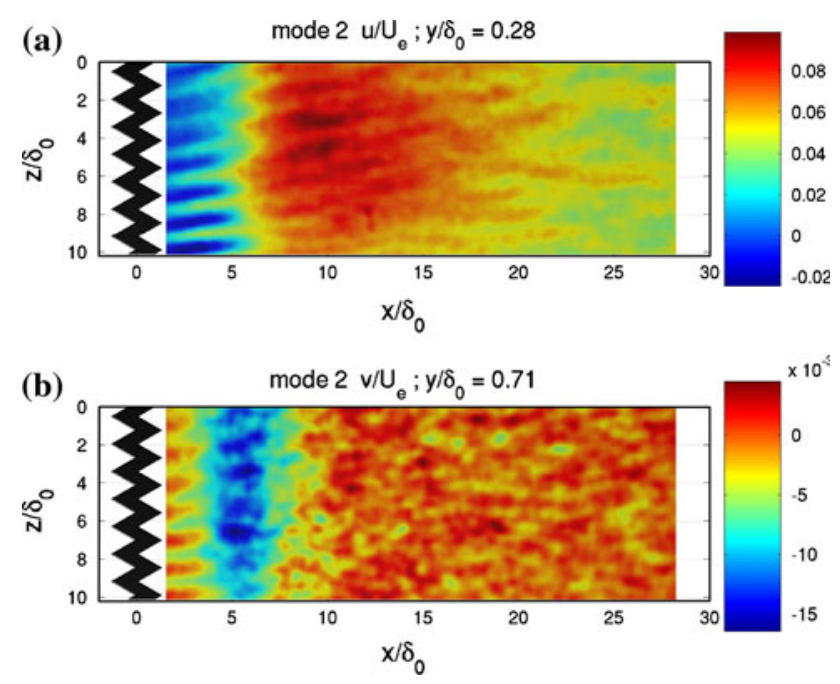

Fig. 13 POD mode 2 associated with a spanwise uniform transport of high momentum fluid toward the wall, $\mathbf{a} u^{\prime}$-component and $\mathbf{b} v^{\prime}$ component in wall-parallel planes $y / \delta_{0}=0.28$ and 0.71 , respectively

Although less energetic, the higher modes contain also large scales. For example, the third POD mode contains nearly spanwise uniform fluctuations near the point of average flow reattachment, similar to the second mode, but not extending as far downstream. Then, the fourth and fifth modes are again linked to the streaks near the trip, as in the first mode.

The results presented here are consistent with those of Erm and Joubert (1991). They also found that downstream of the trip the large flow scales are affected the most, although they attributed the differences to low Reynolds number effects. In particular, the velocity power spectra taken at their measurement location closest to the trip, yet far downstream compared to where the present data were 
taken, showed no collapse and indicated increased energy content at low wave numbers, i.e., large scales. This effect was strongest for the lowest velocity considered, corresponding to the lowest Reynolds number based on trip height. Farther downstream, the power spectra again gave a reasonable collapse, suggesting the flow features introduced by the trip had diminished or disappeared. This occurred after the boundary layer reached a Reynolds number based on momentum thickness of 2,175, which, for the present conditions $\left(U_{e}=0.21 \mathrm{~m} / \mathrm{s}\right)$, is estimated to be $4.6 \mathrm{~m}\left(\sim 1,000 \delta_{0}\right)$ downstream of the trip. Similarly, the dominance of energetic and very slowly decaying largescale flow structures was also observed from the spectra taken downstream of the turbulent reattachment of a laminar separation bubble (Castro and Epik 1998).

\section{Conclusions}

Boundary layer transition behind a zigzag trip was measured using tomographic-PIV revealing the instantaneous three-dimensional velocity distribution and associated flow structures. Although the trip was slightly tilted by approximately 5 degrees with respect to the incoming freestream flow (resulting in a non-zero average spanwise velocity $w$, i.e., cross-flow), the effect on the boundary layer transition is expected to be generally valid. The height of the trip was chosen according to the criterion put forward by Braslow and Knox (1958).

The observed transition scenario suggests zigzag trips may be more efficient with respect to wires in that the spanwise vortices shed from the tripping device already undulate. For a straight wire, a higher Reynolds number based on trip height would likely be required for such spanwise instabilities to develop within the same distance from the trip.

Visualizations of the instantaneous flow provide support for the above conclusion and reveal additional detail. They show a shear layer separating from the trip, which contains undulating spanwise vortices. The corresponding spanwise wavelength is equal to that of the zigzag trip. Underneath the shear layer, backflow is observed with streak structures inside, which appear as elongated regions of spanwise varying $u$-component of velocity. A local minimum in $u$ is found behind each downstream pointing tip of the zigzag, whereas local maxima are located behind upstream pointing tips.

Near the point of average reattachment $\left(x / \delta_{0}=5\right)$, the spanwise vortices break up into arches and subsequently growth legs to form hairpin-like structures. Hairpin packets have also been observed. At this stage, all vortices appear to be of similar size, but as the turbulent boundary layer further develops, new smaller-scale vortical structures are formed close to the wall. The occurrence of these smaller scales coincides with increasing streamwise velocity fluctuations observed in that region, which is traditionally associated with the well know near-wall streaks in turbulent boundary layers.

The mean velocity field is notably very different from the instantaneous snapshots. Instead of spanwise vortices, the mean contains co-rotating streamwise vortices directly behind the trip. The lack of symmetry (that is no counterrotating vortices) is explained by the small tilt of the trip and the resulting cross-flow. The averaging of undulating spanwise vortices convecting downstream causes the streamwise component of the swirling motion (in the legs) to add up in a coherent manner, whereas the wall-normal velocity fluctuations associated with spanwise swirling cancel each other when averaging. It shows that care should be taken when interpreting average velocity fields of transitional and turbulent flows, and that instantaneous 3-D velocity fields, as acquired by tomographic-PIV or instantaneous results from a DNS, are vital when studying the details of transition.

The effect of transition can be noticed downstream as large-scale spanwise coherent motions at least up to the downstream edge of the present measurement domain at $\Delta x / \delta_{0}=28$. These motions are associated with unsteadiness of the separation bubble and separated shear layer directly behind the trip, which both have extended spanwise coherence. The large-scale structure has been observed in both the spanwise autocorrelation function and the energetic POD modes. The latter capture most of the turbulent kinetic energy and hence contribute importantly to the Reynolds stresses.

Acknowledgments Part of the analysis has been performed during participation in the Nordita Turbulent Boundary Layer program at KTH in April 2010. GEE acknowledges the support of a T.U. Delft fellowship.

Open Access This article is distributed under the terms of the Creative Commons Attribution Noncommercial License which permits any noncommercial use, distribution, and reproduction in any medium, provided the original author(s) and source are credited.

\section{References}

Adrian RJ (2007) Hairpin vortex organization in wall turbulence. Phys Fluids 19:041301

Adrian RJ, Westerweel J (2011) Particle image velocimetry. Cambridge University Press, Cambridge

Adrian RJ, Meinhart CD, Tomkins CD (2000) Vortex organization in the outer region of the turbulent boundary layer. J Fluid Mech 422:1-54

Alam M, Sandham ND (2000) Direct numerical simulation of 'short' laminar separation bubbles with turbulent reattachment. J Fluid Mech 403:223-250 
Alving AE, Fernholz HH (1996) Turbulence measurements around a mild separation bubble and downstream of reattachment. J Fluid Mech 322:297-328

Andersson P, Berggren M, Henningson DS (1999) Optimal disturbances and bypass transition in boundary layers. Phys Fluids 11:134-150

Berkooz G, Holmes P, Lumley JL (1993) The proper orthogonal decomposition in the analysis of turbulent flows. Ann Rev Fluid Mech 25:539-575

Boermans LMM (2006) Research on sailplane aerodynamics at Delft University of Technology. Recent and present developments. Netherlands Association of Aeronautical Engineers NVvL, 1 June 2006

Brandt L, Schlatter P, Henningson DS (2004) Transition in boundary layers subject to free-stream turbulence. J Fluid Mech 517:167-198

Braslow AL, Knox EC (1958) Simplified method for determination of critical height of distributed roughness particles for boundarylayer transition at Mach numbers from 0 to 5. NACA TN 4363

Castro IP, Epik E (1998) Boundary layer development after a separated region. J Fluid Mech 374:91-116

Elsinga GE (2008) Tomographic particle image velocimetry and its application to turbulent boundary layers. PhD dissertation, Delft University of Technology, Delft

Elsinga GE, Marusic I (2010) Evolution and lifetimes of flow topology in a turbulent boundary layer. Phys Fluids 22:015102

Elsinga GE, Scarano F, Wieneke B, van Oudheusden BW (2006) Tomographic particle image velocimetry. Exp Fluids 41:933-947

Elsinga GE, Adrian RJ, van Oudheusden BW, Scarano F (2010) Three-dimensional vortex organization in a high-Reynoldsnumber supersonic turbulent boundary layer. J Fluid Mech 644:35-60

Erm LP, Joubert PN (1991) Low-Reynolds-number turbulent boundary layers. J Fluid Mech 230:1-44

Fransson JHM, Brandt L, Talamelli A, Cossu C (2004) Experimental and theoretical investigation of the non-modal growth of steady streaks in a flat plate boundary layer. Phys Fluids 16:3627-3638

Gibbings JC (1959) On boundary layer transition wires. Aeronautical Research Council, CP-462

Hama FR, Long JD, Hegarty JC (1957) On transition from laminar to turbulent flow. J Appl Phys 28:388-394

Humble RA, Scarano F, Van Oudheusden BW (2007) Unsteady flow organization of compressible planar base flows. Phys Fluids 19:076101

Hunt JCR, Wray AA, Moin P (1988) Eddies, stream, and convergence zones in turbulence. Center for Turbulence Report CTR-88, 193-208

Hutchins N, Marusic I (2007) Evidence of very long meandering features in the logarithmic region of turbulent boundary layers. J Fluid Mech 579:1-28

Klebanoff PS (1955) Characteristics of turbulence in a boundary layer with zero pressure gradient. NACA report, No 1247
Klebanoff PS, Tidstrom KD, Sagent LM (1962) The three-dimensional nature of boundary layer instability. J Fluid Mech 12:1-34

Kline SJ, Reynolds WC, Schraub RA, Runstadler PW (1967) The structure of turbulent boundary layers. J Fluid Mech 30:741-773

Lavoie P, Naguib A, Morrison JF (2008) Transient growth induced by surface roughness in a Blasius boundary layer. XXII ICTAM, Adelaide, Australia

Lyon CA, Selig MS, Broeren AP (1997) Boundary layer trips on airfoils at low Reynolds number. 35th AIAA Aerospace Sciences Meeting and Exhibit, Reno, NV, USA, AIAA-97-0511

Perry AE, Lim TT, Teh EW (1981) A visual study of turbulent spots. J Fluid Mech 104:387-405

Preston JH (1958) The minimum Reynolds number for a turbulent boundary layer and the selection of a transition device. J Fluid Mech 3:373-384

Reshotko E (2007) Transition issues for atmospheric entry. 45th AIAA Aerospace Science Meeting and Exhibit, Reno, Nevada, AIAA2007-304

Robinson SK (1991) Coherent motions in the turbulent boundary layer. Ann Rev Fluid Mech 23:601-639

Scarano F, Riethmuller ML (2000) Advances in iterative multigrid PIV image processing. Exp Fluids 29:S051

Schröder A, Geisler R, Staack K, Wieneke B, Elsinga GE, Scarano F, Henning A (2008) Lagrangian and Eulerian views into a turbulent boundary layer flow using time-resolved tomographic-PIV. 14th Int Symp on Appl Laser Techniques to Fluid Mech, Lisbon, Portugal

Sirovich L (1987) Turbulence and the dynamics of coherent structures. Q Appl Math 45:561-590

Smith CR, Walker JDA, Haidari AH, Sobrun U (1991) On the dynamics of near-wall turbulence. Phil Trans R Soc Lond A 336:131-175

Spalart PR, Strelets MK (2000) Mechanisms of transition and heat transfer in a separation bubble. J Fluid Mech 403:329-349

Swearingen JD, Blackwelder RF (1987) The growth and breakdown of streamwise vortices in the presence of a wall. J Fluid Mech 182:255-290

Van Rooij RPJOM, Timmer WA (2003) Roughness sensitivity considerations for thick rotor blade airfoils. J Solar Energy Eng 125:468-478

White FM (1991) Viscous fluid flow, 2nd edn. McGraw-Hill, New York

White EB, Saric WS (2005) Secondary instability of crossflow vortices. J Fluid Mech 525:275-308

Wieneke B (2008) Volume self-calibration for 3D particle image velocimetry. Exp Fluids 45:549-556

Wu X, Moin P (2009) Direct numerical simulation of turbulence in a nominally zero-pressure-gradient flat-plate boundary layer. J Fluid Mech 630:5-41

Zhou J, Adrian RJ, Balachandar S, Kendall TM (1999) Mechanisms for generating coherent packets of hairpin vortices in channel flow. J Fluid Mech 387:353-396 